\title{
Manejo de la ingesta de cuerpos extraños por vía digestiva
}

\section{Management of Foreign bodies ingestion in digestive tract}

José Francisco Cadena-León, ${ }^{1}$ Josefina Monserrat Cázares-Méndez, ${ }^{2}$ Erick Manuel Toro-Monjaraz, ${ }^{3}$ Roberto Cervantes-Bustamante, ${ }^{4}$ Jaime Alfonso Ramírez-Mayans ${ }^{5}$

\section{ANTECEDENTES}

El tratamiento de pacientes pediátricos que de manera accidental ingieren cuerpos extraños es un reto para el medico general, pediatra gastroenterólogo y endoscopista. La Asociación Americana del Centro de Control de Intoxicaciones reportó que $75 \%$ de más de 116,000 casos suceden en menores de 5 años ( 6 meses a 3 años) y $98 \%$ son accidentales. Principalmente se trata de: monedas, juguetes, partes de juguetes, pines, huesos, bolos de comida, joyería, imanes y baterías de botón. ${ }^{1}$

Por lo general, los pacientes son llevados a servicios de urgencias por los padres o cuidadores que evidenciaron la ingestión o tienen sospecha "fundada" de ingestión de un cuerpo extraño, es decir, sin evidenciar el cuidador la ingesta, pero con síntomas sugerentes y factores de riesgo: juguetes u objetos pequeños cerca.

La elección de procedimientos diagnósticos o terapéuticos, y la determinación del pronóstico, dependen de diferentes factores como: tipo de cuerpo extraño ingerido, localización, tiempo de ingestión, síntomas gastrointestinales o respiratorios asociados, disponibilidad de servicios de radiología y endoscopia que cuenten con el equipo e instrumental adecuados, y del personal calificado.

\section{Generalidades}

Los niños pequeños exploran su medio a través de la boca, por lo que la ingesta accidental de un cuerpo extraño es frecuente a esa edad. La mayor parte de los cuerpos extraños ingeridos pueden ser asintomáticos sobre todo en etapas tardías, excepto los objetos grandes, filosos, o las baterías de botón. La recurrencia o ingestión de múltiples objetos es infrecuente y casi siempre sucede en pacientes con trastornos psiquiátricos.

\footnotetext{
${ }^{1}$ Gastroenterólogo pediatra y nutriólogo, endoscopista gastrointestinal, adscrito al Departamento de Gastroenterología y Nutrición, encargado de la Unidad de Endoscopia digestiva.

${ }^{2}$ Adscrita a la unidad de endoscopia digestiva.

${ }^{3}$ Gastroenterólogo pediatra y nutriólogo, adscrito al Departamento de Gastroenterología y Nutrición, encargado del servicio de Motilidad Gastrointestinal.

${ }^{4}$ Gastroenterólogo pediatra y nutriólogo, jefe del Departamento de Gastroenterología y Nutrición.

${ }^{5}$ Gastroenterólogo pediatra y nutriólogo, coordinador de la Unidad Diagnóstica en Gastroenterología Pediátrica Integral, Departamento de Gastroenterología y Nutrición.

Instituto Nacional de Pediatría, Ciudad de México.

Recibido: 13 de febrero 2019

Aceptado: 23 de julio 2019

Correspondencia José Francisco Cadena León pcaden60@hotmail.com

Este artículo debe citarse como Cadena-León JF, Cázares-Méndez JM, Toro-Monjaraz EM, CervantesBustamante R, Ramírez-Mayans JA. Manejo de la ingesta de cuerpos extraños por vía digestiva. Acta Pediatr Mex. 2109;40(5):290-94.
} 
La mayor parte de los cuerpos extraños que pasan al estómago pueden expulsarse espontáneamente a través del tracto gastrointestinal, 10-20\% requieren extracción por vía endoscópica y solamente $1 \%$ mediante un procedimiento quirúrgico. ${ }^{2}$

Los cuerpos extraños que no se expulsan espontáneamente a través del tracto gastrointestinal se impactan en ciertos sitios anatómicos: anillo cricofaríngeo, arco aórtico o esfínter esofágico inferior; en el píloro, ligamento de Treitz, válvula ileocecal o en la unión rectosigmoidea; o en pacientes con ciertos padecimientos: estenosis esofágica, acalasia, divertículo de Meckel, o con bridas abdominales que condicionan un sitio de estrechez fisiológica o patológica. Desde luego que deben descartarse otras afecciones asociadas inflamatorias: esofagitis eosinofílica.

Las manifestaciones clínicas dependerán del tipo de objeto, localización, edad del paciente y el tiempo de ingestión.

\section{Diagnóstico y tratamiento}

El diagnóstico del paciente que ha ingerido un cuerpo extraño se inicia con la historia clínica detallada que nos permita identificar el tipo de objeto y tiempo trascurrido desde la ingestión, síntomas asociados, adecuada exploración física: estado ventilatorio, estabilidad cardiovascular, datos de obstrucción o perforación y estado neurológico.

El estudio radiológico está indicado en todos los pacientes con sospecha o ingesta documentada de cuerpo extraño. Las proyecciones anteroposterior y lateral permiten la identificación, localización y naturaleza de cuerpos extraños radioopacos (metálico 100\%, cristal 43\%, huesos de pescado $26 \%$ ), monedas, pilas de botón (signo de doble halo y step off), en caso de cuerpos extraños radiolúcidos signos de complicaciones: concentraciones hidroaéreas, perforación de la vía gastrointestinal ${ }^{2}$
La videoendoscopia flexible es un procedimiento invasivo diagnóstico-terapéutico que permite, a través de un tubo flexible acompañado de un sistema de video y captura de imágenes, la visualización del tubo digestivo, toma de biopsias y procedimientos de extracción de cuerpos extraños, dilataciones de estenosis y otros procedimientos.

Las indicaciones de la extracción de cuerpos extraños por endoscopia digestiva dependerán del tipo de cuerpo extraño, edad del paciente, localización, tiempo de ingestión, ayuno, disponibilidad de equipo e instrumental, y personal calificado. Es importante evitar procedimientos innecesarios en turnos nocturnos, con poco personal o falta de médicos endoscopistas con mayor experiencia. ${ }^{3}$

El tiempo de extracción endoscópica se define según el tipo de cuerpo extraño y localización, síntomas y riesgos de complicaciones (aspiración, obstrucción o perforación):

a. Emergencia: menos de 2 horas de la ingesta de cuerpo extraño, sin ayuno.

b. Urgencia: menos de $24 \mathrm{~h}$ de la ingesta de cuerpo extraño, ayuno.

c. Electivo: más de 24 horas, ayuno.

Los videogastroscopios flexibles ofrecen mayor seguridad y eficacia comparada con otros métodos de extracción: endoscopios rígidos, sondas de Foley y otros que dependen de la experiencia del médico y lugar donde se realiza. Permite utilizar diferentes instrumentos para la extracción de cuerpos extraños: asas de polipectomía, canastillas de cuerpos extraños, pinzas con dientes de ratón, de cocodrilo, trípode, uso de "capuchones" o "sobretubos" para reducir lesiones en casos de cuerpos extraños punzocortantes, visión de lesiones mucosas y toma de biopsias en casos necesarios. ${ }^{3}$ El procedimiento quirúrgico está indicado en cuerpos extraños en los que no es posible llevar a cabo el estudio 
endoscópico con un alto riesgo de perforación: imanes (2 o más), objetos puntiformes, pilas de botón localizadas en intestino medio, sin movimiento, con controles radiográficos.

\section{TIPOS DE OBJETO}

\section{Monedas}

Es el cuerpo extraño ingerido con más frecuencia por los niños, $70 \%$ se localiza en el tercio superior, $20 \%$ en el tercio medio y $10 \%$ en el tercio inferior. ${ }^{4}$ El paso espontáneo ocurre en $30 \%$ de los pacientes (8-16 h). Las monedas mayores de $23.5 \mathrm{~mm}$ de diámetro tienen mayor riesgo de impactarse en niños menores de 5 años. ${ }^{5}$ Cuando se retienen en el esófago durante un tiempo desconocido, o más de 24 horas, lo indicado es la extracción.

\section{Baterías de botón}

La ingesta accidental de baterías de botón es una urgencia por la posibilidad de generar quemaduras, hemorragias y necrosis por licuefacción por contacto de los dos polos con la mucosa y fuga de álcalis y metales pesados: mercurio, plata, litio hidróxido de sodio y potasio que pueden originar perforaciones, estenosis y fístulas en la vía gastrointestinal. El riesgo es mayor con baterías de más de 20 mm de diámetro y que contienen litio. ${ }^{6,7}$

\section{Objetos punzo-cortantes}

Los objetos más frecuentes son: pines, agujas, espinas de pescado, huesos de pollo, palillos de dientes de madera, aretes, seguros de ropa de bebé, con riesgo de perforación en 10-35\% cuando se localizan en la hipofaringe y la región ileocecal. Originan síntomas de odinofagia, disfagia, y complicaciones: perforación, migración extraluminal, abscesos retrofaríngeos, edema en el cuello, crépitos o neumomediastino. La erosión hacia la aorta se asocia con sangrado digestivo severo. ${ }^{6,7}$
Imanes

Son un problema de salud emergente en virtud del aumento en la potencia mayor de 10 veces que la de los imanes convencionales, mayor disponibilidad en artículos de oficina, juegos de niños, juegos antiestrés que se componen de 200 o más estructuras con formas diferentes: cubos, bolas magnéticas o cilindros. Las complicaciones se presentan con la ingestión de dos o más imanes o un imán con un objeto metálico que son capaces de alinearse entre dos asas intestinales produciendo una imagen de "objeto único" y atrapamiento de asas intestinales con necrosis, perforación y fístulas. Cuando se ingiere un imán se recomienda la vigilancia del paso a través del tubo digestivo, en casos de dos o más imanes o un objeto metálico, lo indicado es la extracción endoscópica. ${ }^{8}$

\section{Objetos largos}

Los cuerpos extraños con diámetro mayor de $25 \mathrm{~mm}$ y $6 \mathrm{~cm}$ de longitud no pasan a través del píloro en $80 \%$ de los casos. El $50 \%$ de los cuerpos extraños de 5-6 cm se impacta en la región íleocecal: cepillos de dientes, cucharas o pilas. Los riesgos dependen de la localización y forma del objeto. ${ }^{9,10}$

Otros cuerpos extraños son la videocápsula endoscópica para el diagnóstico de enfermedad de Crohn en 1.5-3.5\% de los casos con impactación esofágica por alimentos, donde deben sospecharse alteraciones motoras de esófago, anillos (Schatzky) estenosis, esofagitis eosinofílica o enfermedad por reflujo gastroesofágico. Los polímeros superabsorbentes (PSabs), que son capaces de aumentar su volumen 30-60 veces, ocasionan obstrucción intestinal. Se considera una urgencia y los bezoares: fórmulas infantiles (lactobezoares) cabello (tricobeozares) y medicamentos (farmacobeozares) que son infrecuentes.

\section{Figura 1}




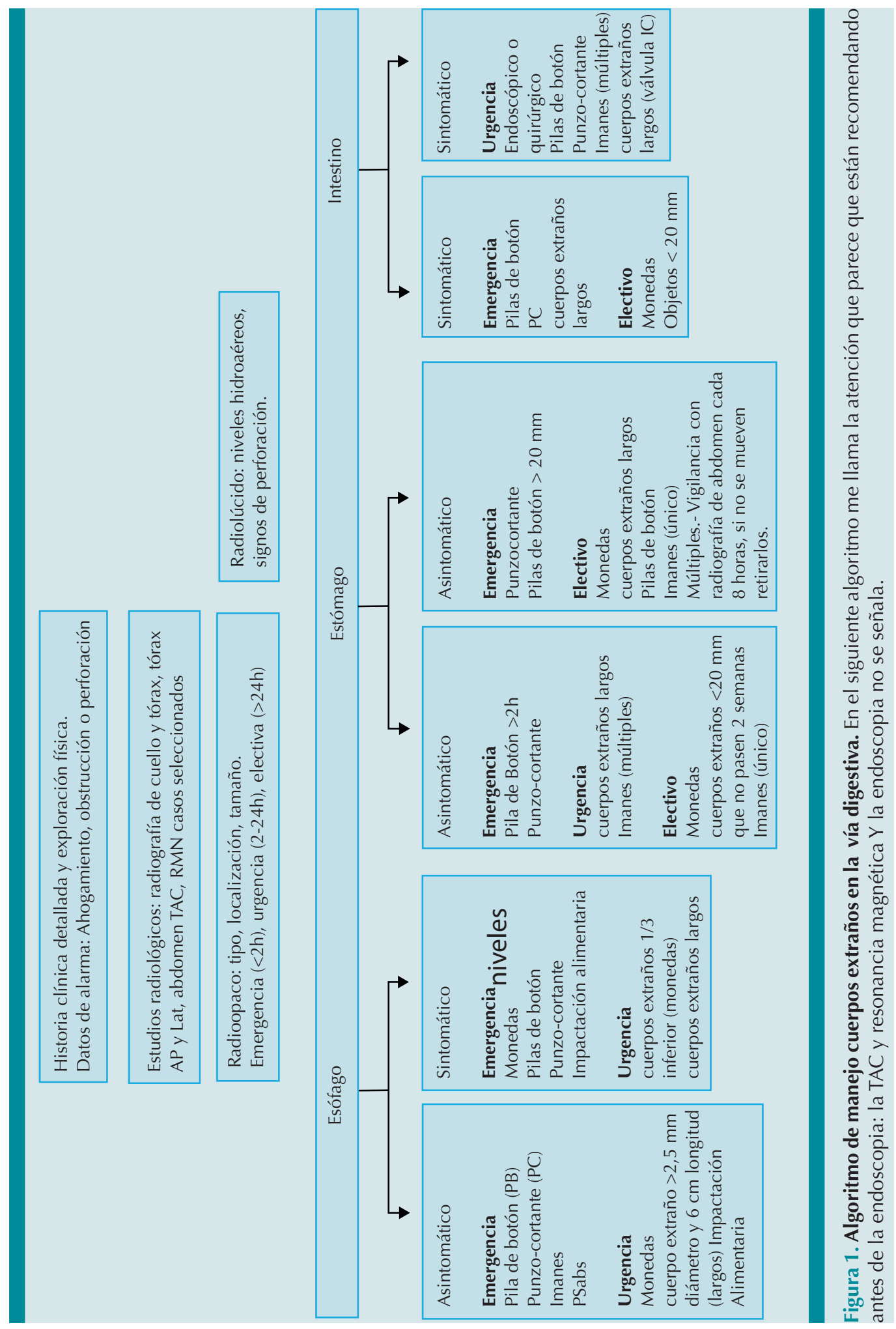




\section{CONCLUSIONES}

El abordaje diagnóstico de la ingestión de cuerpos extraños son un reto para los médicos generales, pediatras y otros especialistas a la hora de tomar decisiones para la extracción endoscópica. El conocimiento de los diferentes tipos de cuerpo extraño, localización y síntomas permite establecer, en la mayoría de los casos, la conducta diagnóstica-terapéutica. Los cuerpos extraños puntiformes, pilas de botón, monedas en la parte superior del esófago sintomáticas representan una emergencia y siempre deben extraerse lo más pronto posible.

\section{REFERENCIAS}

4. Kramer RE. Management of Ingested Foreign Bodies in Children: A clinical Report of the NASPGHAN Endoscopy Committee. J Pediatr Gastroenterol Nutr. 2015;60:562-74. doi: 10.1097/MPG.0000000000000729

5. Gilger MA, Jain AK. Foreign bodies of the esophagus and gastrointestinal tract in children. Upto Date Feb 2018 https://www.uptodate.com/contents/foreign-bodies-ofthe-esophagus-and-gastrointestinal-tract-in-children

6. Singh B, et al. Endoscopic management of ingested foreign bodies and food impaction in esophagus. J Dig Endosc. 2015; 6:96-100. doi: 10.4103/0976-5042.165689

7. Kurowski JA, Kay M. Caustic ingestion and Foreign Bodies Ingestions in Pediatric patients. Pediatr Clin N Am. 2017;(64):507-524. https://doi.org/10.1016/j. pcl.2017.01.004

8. Tander B, Yazici M, Rizalar R, et al. Coin ingestion in children; wich size is more risky? J Laparoendosc Adv Surg Tech A. 2009;19:241-3.

9. Wright C.C, Closson FT. Updates in Pediatric Gastrointestinal Foreign Bodies. Pediatr Clin N Am. 2013:1-19

10. Uyemura MC. Foreign body ingestion in children. AM Fam Physician. 2005;72(2)287-91.

11. Cadena León J, Cázares Méndez M, Arguello Bermeo R, Cervantes Bustamante R, Ramírez Mayans J. Ingestión accidental de imanes: Un problema de salud emergente. Rev Gastroenterol Mexico. 2015;80:113-15.

12. Carp L. Foreign Bodies in the intestine. An surg. 1927;85:575-91.

13. Eisen $\mathrm{GM}$, et al. Guideline for the management of ingested foreign bodies. Gastrointest Endosc. 2002;55:802. https:// doi.org/10.1016/S0016-5107(02)70407-0 